\title{
Prevalencia del riesgo de desnutrición y situación de la terapia nutricional en pacientes adultos hospitalizados en Perú
}

\author{
Prevalence of malnutrition risk and nutritional therapy situation in adult \\ hospitalized patients in Peru \\ Prevalência do risco de desnutrição e situação da terapia nutricional em pacientes adultos \\ internados no Peru
}

Brian Wally Mariños Cotrina ${ }^{1 *}$, Roxana Esther Segovia Denegri², Edinson Joel Arévalo Cadillo, Melissa Ponce Castillo ${ }^{4}$, Paulo César Arias De la Torre ${ }^{5}$, Diana Antonia Ponce Castillo ${ }^{6}$, Camila Rossana Muñoz Carpio ${ }^{7}$, Daniel Fernando Mendez Carbajal ${ }^{7}$, Carlos Alfredo Galindo Martins ${ }^{8}$

Recibido: 13 de noviembre 2019. Aceptado para publicación: 4 de marzo 2020.

Publicado en línea: 11 de abril de 2020

https://doi.org/10.35454/rncm.v3n2.28

\begin{abstract}
Resumen
Introducción: la desnutrición intrahospitalaria sigue siendo un gran problema en la actualidad alcanzando una prevalencia de 50,2 \%, según el Estudio Latinoamericano de Desnutrición (ELAN). Dado que en Perú no hay cifras reportadas, el presente estudio tiene el objetivo de determinar la prevalencia del riesgo de desnutrición y la situación de la terapia nutricional en pacientes adultos hospitalizados de enero a marzo de 2019.

Método: estudio de tipo descriptivo observacional de corte transversal. Se evaluó el riesgo de desnutrición en pacientes adultos hospitalizados de los servicios de medicina, unidad de cuidados intensivos (UCI) y cirugía en once centros hospitalarios del territorio peruano utilizando la herramienta de tamizaje nutricional (NRS2002) dentro de las 48 horas de su ingreso.

Resultados: de 1.731 pacientes incluidos, 50,3\% eran mujeres, la edad media de $56 \pm 21$ años. La prevalencia de pacientes nutricionalmente "en riesgo" fue $69,7 \%$, siendo más alta en pacientes quirúrgicos $(29,3 \%)$ y cuidados intensivos $(15,4 \%)$, y más baja en pacientes con enfermedades hematológicas (1,7\%). El tipo de terapia nutricional más usada en pacientes con
\end{abstract}

\section{Abstract}

Background: In-hospital malnutrition continues to be a major problem today, reaching a prevalence of $50.2 \%$, according to the Latin American Study of Malnutrition (ELAN). Since there are no reported figures in Peru, the present study aims to determine the prevalence of the risk of malnutrition and the status of nutritional support in adult patients hospitalized from January to march 2019.

Methodology: Cross-sectional descriptive study conducted in eleven hospitals in Peru. The risk of malnutrition was evaluated in hospitalized adult patients of the medical services, intensive care unit (ICU) and surgery in eleven hospitals using the nutritional screening tool (NRS-2002) within 48 hours of its entry.

Results: Of 1731 patients included, $50.3 \%$ were women, mean age of $56 \pm 21$ years. The prevalence of nutritionally "at risk" patients was $69.7 \%$. The prevalence of patients at nutritional risk was higher in surgical (29.3\%) and intensive (15.4\%) patients and lower in patients with hematological diseases (1.7\%). The type of nutritional support therapy most used in patients with nutritional risk was the oral route or artisanal formula (42.3\%) followed

\section{Resumo}

Introdução: a desnutrição hospitalar continua sendo um grande problema hoje, atingindo uma prevalência de 50,2 \%, segundo o Estudo Latino-Americano de Desnutrição (ELAN). Dado que no Peru não há dados registados, o presente estudo tem como objetivo determinar a prevalência do risco de desnutrição e a situação da terapia nutricional em pacientes adultos hospitalizados de janeiro a março de 2019.

Método: estudo de tipo descritivo observacional transversal. O risco de desnutrição foi avaliado em pacientes adultos hospitalizados dos serviços de medicina, unidade de cuidados intensivos (UCI) e cirurgia em onze centros hospitalares no território peruano, utilizando a ferramenta de triagem nutricional (NRS-2002) nas 48 horas após o seu ingresso.

Resultados: dos 1.731 pacientes incluídos, 50,3 \% eram mulheres, a idade média foi de $56 \pm 21$ anos. A prevalência de pacientes nutricionalmente "em risco" foi de $69,7 \%$, sendo maior nos pacientes cirúrgicos (29,3\%) em cuidados intensivos $(15,4 \%)$ e menor nos pacientes com doenças hematológicas (1,7\%). $\mathrm{O}$ tipo de terapia nutricional mais utilizada em pa- 
riesgo nutricional fue la vía oral o fórmula artesanal $(42,3 \%)$ seguida de ayuno con $32,4 \%$, la vía parenteral complementaria no se consideró una elección.

Conclusiones: en Perú, el riesgo nutricional en el ámbito hospitalario es una condición prevalente muy alta. Estar nutricionalmente "en riesgo" afecta a siete de cada diez pacientes. Además, se sugiere que la provisión de la terapia de apoyo nutricional complementaria es crucial en esta condición, por ello la consolidación del trabajo en equipos multiprofesionales y la identificación e intervención temprana del riesgo nutricional son imperativas.

Palabras clave: hospital, desnutrición, estado nutricional, nutrición enteral, nutrición parenteral, dietoterapia, ayuno. by fasting with $32.4 \%$, while the complementary parenteral route was not a choice.

Conclusions: In Peru, nutritional risk in the hospital is a highly prevalent condition. Being nutritionally "at risk" affects seven out of ten patients. In addition, it is suggested that the provision of complementary nutritional support therapy is crucial in this condition, which is why the multidisciplinary consolidation and early identification and intervention of nutritional risk are imperative.

Keywords: Hospital; Malnutrition; Nutritional status; Enteral nutrition; Parenteral nutrition; Nutrition therapy; Fasting cientes com risco nutricional foi a via oral ou a fórmula artesanal (42,3\%) seguida de jejum com $32,4 \%$, enquanto a via parenteral complementar não foi uma escolha.

Conclusões: no Perú, o risco nutricional no ambiente hospitalar é uma condição de muito alta prevalência. Estar nutricionalmente "em risco" afeta sete em cada dez pacientes. Além disso, sugere-se que o fornecimento de terapia nutricional complementar seja crucial nesta condição, portanto, a consolidação do trabalho em equipa multiprofissional e a identificação e intervenção precoce no risco nutricional são imperativas.

Palavras-chave: hospital, desnutrição, estado nutricional, nutrição enteral, nutrição parenteral, dietoterapia, jejum.

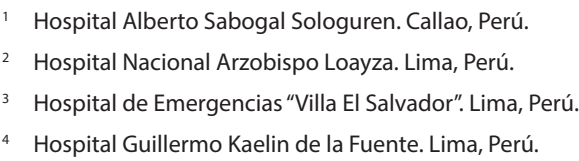

\section{INTRODUCCIÓN}

La desnutrición hospitalaria es un problema de salud pública en el mundo. De acuerdo con estudios internacionales su prevalencia se encuentra entre $20 \%$ y $50 \%$, pudiendo llegar a $80 \%$ en adultos mayores y pacientes oncológicos, mientras que en unidades de emergencias puede llegar a más de $60 \%^{(1,2)}$.

Diferentes estudios han demostrado que la desnutrición hospitalaria se asocia a un incremento en el riesgo de complicaciones, estancias hospitalarias más prolongadas, pronóstico negativo y mortalidad, ello implica entonces, el aumento de los costos de atención al paciente ${ }^{(3,4)}$.

Es así que el tamizaje nutricional es una herramienta que permite identificar el riesgo de desnutrición dentro del ámbito hospitalario, de forma fácil y efectiva ${ }^{(5)}$. La Sociedad Europea de Nutrición Clínica y Metabolismo (ESPEN) lo define como un proceso rápido y simple realizado por el personal de salud al ingreso al hospital. Su propósito es predecir la probabilidad de un mejor o peor resultado debido a factores nutricionales y en la manera en la que el tratamiento nutricional puede influir en este ${ }^{(6)}$.

\footnotetext{
5 Hospital de Emergencias “José Casimiro Ulloa". Lima, Perú.

6 Hospital Nacional Dos de Mayo. Lima, Perú.

7 Universidad Nacional Federico Villarreal. Lima, Perú

8 Hospital San Ángel INN. Ciudad de México, México.
}

Sin bien, actualmente, existen diversas herramientas de tamizaje ${ }^{(7)}$ en esta investigación aplicamos el Nutrition Risk Score 2002 (NRS-2002) debido a que está recomendada por ESPEN para la identificación de riesgo nutricional en pacientes hospitalizados ${ }^{(8)}$. Además se destaca por tener un gran valor pronóstico, buena relación con los parámetros antropométricos, bioquímicos y predicción de mortalidad en comparación con otras herramientas de tamizaje ${ }^{(9-12)}$.

Debido a la importancia de la identificación temprana del riesgo nutricional y al vacío de información en la población peruana, se realizó el presente estudio con el objetivo de identificar el riesgo de desnutrición, diagnóstico médico más relacionado con el riesgo nutricional y tipo de apoyo nutricional más utilizado en pacientes adultos peruanos al ingreso hospitalario.

\section{MATERIALES Y METODOLOGÍA}

\section{Diseño del estudio}

Estudio de tipo descriptivo observacional de corte transversal el cual involucró a 11 centros hospitalarios 
del territorio nacional. La información del paciente se obtuvo durante las primeras 48 horas de ingreso a los servicios de hospitalización quirúrgica, medicina interna, y cuidados intensivos, entre otros.

\section{Centros hospitalarios}

Se eligieron los centros hospitalarios por conveniencia a través de una convocatoria nacional gremial. Como resultado, se incluyeron 11 centros hospitalarios del territorio peruano. Los centros debían contar con servicios de hospitalización de por lo menos nivel II de complejidad, servicio de nutrición y licenciados en Nutrición como parte del equipo multidisciplinario de atención hospitalaria. Además, se excluyeron servicios policlínicos, centros de salud o puestos de salud.

\section{Participantes del estudio}

Este estudio involucró 1.731 pacientes. Se incluyeron adultos hospitalizados de ambos géneros con estancias hospitalarias $<48$ horas. Se excluyeron gestantes, puérperas, pacientes con alteraciones genéticas y pediátricas, ambulatorios y domiciliarios.

\section{Recolección de datos}

De la historia clínica se obtuvieron datos de variables demográficas como sexo, edad y centro hospitalario de procedencia; las edades fueron agrupadas en $<40$ años, 40 - 60 años y $\geq 60$ años. El diagnóstico médico principal se consideró variable médica, el cual fue obtenido de la historia clínica y posteriormente registrado en una ficha o encuesta. Para determinar el riesgo nutricional se aplicó la herramienta de tamizaje NRS 2002, en donde se consideró "con riesgo nutricional" a los pacientes con un puntaje $\geq 3 \mathrm{y}$ "sin riesgo nutricional" a aquellos con puntaje $<3^{(6,7)}$. Se recolectaron datos referentes al tipo de terapia nutricional recibida (nada por vía oral, alimentos vía oral o enteral artesanal, suplementos nutricionales orales, nutrición enteral, nutrición parenteral total, nutrición parenteral complementaria).

\section{Análisis estadístico}

Se realizó un análisis descriptivo en el cual se emplearon frecuencias y porcentajes en variables cualitativas, promedios y desviaciones estándar en aquellas cuantitativas.

\section{Consideraciones éticas}

El estudio se ajusta a la normativa ética nacional y sigue los principios éticos de la Declaración de Helsinki. Además, este estudio se conceptualiza como una investigación sin riesgo debido a que solo se recolectaron datos relacionados con cada uno de los sujetos sin ponerlos en situación de riesgo.

\section{RESULTADOS}

Se incluyeron 1.731 pacientes procedentes de 11 centros hospitalarios públicos de diferentes regiones del Perú y de distintos niveles de atención. El 58,8 \% de los datos aportados son de hospitales de segundo nivel de atención ( 6 hospitales) y 42,2 \% del tercer nivel de atención ( 5 hospitales). Las características de cada centro se muestran en la Tabla 1.

Tabla 1. Características de los centros hospitalarios

\begin{tabular}{|c|c|c|c|c|}
\hline $\begin{array}{c}\text { Código } \\
\text { del centro } \\
\text { hospitalario }\end{array}$ & $\begin{array}{c}\text { Nivel de } \\
\text { atención }\end{array}$ & $\begin{array}{c}\text { Cate- } \\
\text { goría }\end{array}$ & $\begin{array}{c}\text { Pacientes } \\
\text { incluidos } \\
\text { (n) }\end{array}$ & $\begin{array}{c}\text { Porcentaje } \\
\text { del total } \\
\text { (\%) }\end{array}$ \\
\hline $\mathbf{1}$ & Segundo & II-2 & 55 & 3,18 \\
\hline $\mathbf{2}$ & Segundo & II-E & 50 & 2,89 \\
\hline $\mathbf{3}$ & Segundo & II-E & 59 & 3,41 \\
\hline $\mathbf{4}$ & Segundo & II-E & 553 & 31,95 \\
\hline $\mathbf{5}$ & Tercer & III-1 & 140 & 8,09 \\
\hline $\mathbf{6}$ & Tercer & III-1 & 149 & 8,61 \\
\hline $\mathbf{7}$ & Tercer & III-E & 159 & 9,19 \\
\hline $\mathbf{8}$ & Segundo & II-1 & 105 & 6,07 \\
\hline $\mathbf{9}$ & Segundo & II-2 & 196 & 11,32 \\
\hline $\mathbf{1 0}$ & Tercer & III-I & 212 & 12,25 \\
\hline $\mathbf{1 1}$ & Tercer & III-2 & 53 & 3,06 \\
\hline
\end{tabular}

II-1: Hospital I, II-2: Hospital II, II-E: Hospital 2 Especializado, III-1: Hospital III, III-2: Instituto Especializado, III-E: Hospital III Especializado.

Del total de pacientes, la mayoría fue de sexo femenino con 50,3\% ( $\mathrm{n}=870)$, la edad promedio fue $56 \pm$ 21 años, la proporción por grupos de edad fue $26,1 \%$ para $<40$ años, $27,4 \%$ para 40 a 60 años y $46,5 \%$ para $\geq 60$ años. El tipo de diagnóstico médico más frecuente fue el "quirúrgico" (29,3\%) seguido de "pacientes críticos" (15,4\%). El riesgo nutricional se encontró en casi $70 \%$ de los pacientes evaluados, analizando la 
población por tipo de diagnóstico médico principal, los pacientes en estado crítico presentaron $97 \%$ de riesgo seguido de los pacientes quirúrgicos $(87,4 \%)$ y enfermedad cardiovascular $(73,5 \%)$. En pacientes oncológicos se encontró $44,4 \%$ de riesgo nutricional, como puede observarse en la Tabla 2.

Tabla 2. Diagnóstico médico principal de pacientes según el riesgo nutricional

\begin{tabular}{|l|c|c|c|c|c|c|}
\hline \multirow{2}{*}{$\begin{array}{c}\text { Diagnóstico } \\
\text { médico } \\
\text { principal }\end{array}$} & \multicolumn{3}{|c|}{ Riesgo nutricional } & \multicolumn{2}{c|}{ Total } \\
\cline { 2 - 6 } & $\begin{array}{c}\text { Sin riesgo } \\
\text { nutricional }\end{array}$ & \multicolumn{2}{c|}{$\begin{array}{c}\text { Con riesgo } \\
\text { nutricional }\end{array}$} & \multicolumn{2}{|}{} \\
\cline { 2 - 6 } & $\mathbf{n}$ & $\%$ & $\mathbf{n}$ & $\%$ & $\mathbf{n}$ & $\%$ \\
\hline $\begin{array}{l}\text { Sistema } \\
\text { locomotor }\end{array}$ & 62 & 63,3 & 36 & 36,7 & 98 & 5,7 \\
\hline Cardiovascular & 18 & 26,5 & 50 & 73,5 & 68 & 3,9 \\
\hline Digestivo & 121 & 47,5 & 134 & 52,5 & 255 & 14,7 \\
\hline Endocrinológico & 47 & 51,1 & 45 & 48,9 & 92 & 5,3 \\
\hline Genitourinario & 31 & 58,5 & 22 & 41,5 & 53 & 3,1 \\
\hline Renal & 23 & 39,0 & 36 & 61,0 & 59 & 3,4 \\
\hline Hematológico & 8 & 38,1 & 13 & 61,9 & 21 & 1,2 \\
\hline Oncológico & 15 & 55,6 & 12 & 44,4 & 27 & 1,6 \\
\hline Neurológico & 33 & 45,8 & 39 & 54,2 & 72 & 4,2 \\
\hline Respiratorio & 41 & 30,8 & 92 & 69,2 & 133 & 7,7 \\
\hline Quirúrgico & 64 & 12,6 & 443 & 87,4 & 507 & 29,3 \\
\hline Estado crítico & 8 & 3,0 & 259 & 97,0 & 267 & 15,4 \\
\hline No disponible & 53 & 67,1 & 26 & 32,9 & 79 & 4,6 \\
\hline Totales & $\mathbf{5 2 4}$ & $\mathbf{3 0}$ & $\mathbf{1 2 0 7}$ & $\mathbf{7 0}$ & $\mathbf{1 7 3 1}$ & $\mathbf{1 0 0}$ \\
\hline
\end{tabular}

De acuerdo con el tipo de soporte nutricional, 42,3\% de los sujetos con riesgo nutricional recibieron alimentos por vía oral o a través de una fórmula enteral artesanal, seguido de pacientes que se encontraban al momento de la evaluación en estado de ayuno $(32,4 \%)$ y de aquellos con nutrición enteral a través de una fórmula industrializada (16,5\%). Cabe mencionar que en 368 pacientes no se obtuvo información respecto al tipo de nutrición recibida, por lo que para este análisis se trabajó con una muestra de 1.363 . Ningún paciente en riesgo nutricional recibió nutrición parenteral complementaria en presencia de una nutrición enteral insuficiente (Tabla 3).
Tabla 3. Tipo de terapia nutricional en pacientes según el riesgo nutricional

\begin{tabular}{|l|c|c|c|c|}
\hline \multirow{2}{*}{ Tipo de terapia nutricional } & \multicolumn{4}{|c|}{ Riesgo nutricional } \\
\cline { 2 - 5 } & $\begin{array}{c}\text { Sin riesgo } \\
\text { nutricional }\end{array}$ & \multicolumn{2}{c|}{$\begin{array}{c}\text { Con riesgo } \\
\text { nutricional }\end{array}$} \\
\cline { 2 - 5 } & $\mathbf{n}$ & $\%$ & $\mathbf{n}$ & $\%$ \\
\hline Nada por vía oral & 19 & 4,10 & 291 & 32,40 \\
\hline $\begin{array}{l}\text { Alimentos vía oral o enteral } \\
\text { artesanal }\end{array}$ & 434 & 93,30 & 380 & 42,30 \\
\hline $\begin{array}{l}\text { Suplementos nutricionales } \\
\text { orales }\end{array}$ & 10 & 2,20 & 79 & 8,80 \\
\hline Nutrición enteral & 1 & 0,20 & 148 & 16,50 \\
\hline Nutrición parenteral total & 1 & 0,20 & 0 & 0,00 \\
\hline $\begin{array}{l}\text { Nutrición parenteral } \\
\text { complementaria }\end{array}$ & 0 & 0,00 & 0 & 0,00 \\
\hline
\end{tabular}

\section{DISCUSIÓN}

La prevalencia de riesgo de desnutrición observada en el presente estudio, utilizando la herramienta de tamizaje NRS2002, fue aproximadamente $70 \%$ en adultos hospitalizados en Perú, lo cual revela una situación de salud pública alarmante.

Esta cifra es mayor a las reportadas con anterioridad en nuestro país ${ }^{(13,14)}$, que se acercan a $57 \%$, usando la misma herramienta de tamizaje. Carrasco, et al. en un estudio realizado en un hospital general en la ciudad de Lima, encontraron una prevalencia de riesgo nutricional en pacientes adultos del servicio de medicina de $56,7 \%$, cifra ligeramente más baja a la reportada por Arroyo-Sánchez et al. en un hospital de Trujillo (58\%), pero en ambos casos menor a la encontrada en el presente estudio: $70,3 \%$ lo que se correlaciona con mayores tasas de reingreso y mortali$\mathrm{dad}^{(15)}$ y que probablemente refleje mejor la situación actual de nuestro país por ser el primer estudio de mayor alcance nacional.

Por otro lado, se reportó riesgo nutricional en $44 \%$ de los pacientes oncológicos, cifra menor a la informada por Zhang et al. $(69,5 \%)$ en China ${ }^{(16)} y$ por Alvarez-Altamirano et al. $(50,2 \%)$ en México ${ }^{(17)}$. Escenarios que difieren con el estudio de Carrasco et al. realizado en Lima en 2015 donde todos los pacientes con neoplasia de un nosocomio presentaron riesgo nutricional $^{(13)}$. Situación que, probablemente, ha disminuido en los últimos años por las mejoras en las tasas 
de detección de cáncer precoz en nuestro país y que se asemejan a otros países.

Diferentes autores han descrito la relación existente entre el diagnóstico médico y el riesgo nutricional ${ }^{(18-23)}$. El presente estudio encontró que la prevalencia de riesgo nutricional fue más frecuente en pacientes quirúrgicos con $87,4 \%$ y en estado crítico con $97 \%$, situación que coincide con otros estudios que informan tasas entre $50 \%$ y $60 \%{ }^{(15,24,25)}$. Esto probablemente se deba a que las características particulares de las condiciones metabólicas tienen un impacto mayor de desarrollar desnutrición; además de estar asociada al aumento de la estancia hospitalaria, costos y resultados clínicos adversos.

La terapia nutricional en el paciente hospitalizado es crucial para cubrir sus requerimientos y prevenir la desnutrición, debido a su asociación a mortalidad, complicaciones infecciosas y costos de hospitalización ${ }^{(20,26,27)}$. En el presente estudio, cerca de la mitad de los pacientes con riesgo nutricional (47\%) recibieron alimentación oral y enteral artesanal, mientras que la vía parenteral no fue una elección en la nutrición del paciente, puesto que su utilización es menor de $1 \%$ si nos referimos a nutrición parenteral total y complementaria. Esto concuerda con lo descrito en otro estudio realizado en Latinoamérica, donde se observó el bajo porcentaje de nutrición parenteral total y complementaria en presencia de nutrición enteral insuficiente, con 9,4 \% y 10,7 \% respectivamente ${ }^{(28,29)}$. Esto puede deberse a las controversias que aún existen respecto a la nutrición parenteral y a la brecha entre las pautas y la práctica clínica diaria que pueden diferir entre hospitales ${ }^{(30,31)}$.

Es la primera vez que en Perú se describe la práctica nutricional de sus centros hospitalarios, y de más preponderancia aún es la inclusión de 11 nosocomios de distintas regiones y niveles de atención. Como limitante del estudio está la inclusión de pacientes con diferentes estadios o severidad de enfermedades, lo cual no se realizó en la presente investigación y debería ser considerado en futuras investigaciones. Cabe mencionar aquí otra limitante encontrada y es que los hospitales involucrados tenían mínimo nivel II y tenían un nutricionista, por lo que se desconoce si en hospitales de menor complejidad y sin nutricionista la situación podría ser drásticamente diferente.

\section{CONCLUSIÓN}

El riesgo nutricional es una condición prevalente en los hospitales evaluados en el territorio peruano. La presente investigación establece que 7 de cada 10 sujetos se encuentran en riesgo de desnutrición y que el diagnóstico quirúrgico es el que más se relaciona con esta situación. Por tal motivo, se exhorta a la consolidación del trabajo en equipos multidisciplinarios, donde el profesional nutricionista participa en el proceso de cuidado nutricional con la finalidad de brindar información individualizada para el apoyo al tratamiento integral.

\section{Agradecimientos}

A nuestros colaboradores y personal asistencial que permitieron el desarrollo de esta investigación: Cinthia Mónica Zeballos Alcalde, María Luz Cueva Rossell, Isabel Pineda Vásquez, Mercedes Haidee Argomedo Muñoz, Jessica Camac Román, Marleydi Nuñez Aropaza, Eva Huanca Maquera, Edinson Sánchez Sánchez, Diana Quispe Arbildo, Zoila Filomena Paredes Tarazona, Beatriz Gutierrez Cuti, Teresa Irribarren León, Teresa Dominga Lajo, Crhisty Fiorella Guerra Cáceres, Olimpia Ramos Lupaca y Roxana Zoila Román Gameros y Esteban Jurado Beizaga.

\section{Financiación}

Ninguna entidad particular financió la realización del presente estudio.

\section{Conflicto de intereses}

Los autores declaran no tener conflicto de interés alguno.

\section{Declaración de autoría}

PAT y EJAC participaron en la concepción del estudio y análisis de los resultados; BWMC participó en la obtención y consolidación de los datos; CAGM participó en el análisis de los datos. Todos los autores revisaron el artículo y validaron su versión final.

\section{Referencias bibliográficas}

1. Raupp D, Silva FM, Marcadenti A, Rabito EI, da Silva Fink J, Becher P, et al. Nutrition screening in public hospital emergency rooms: Malnutrition universal screening tool and Nutritional Risk Screening-2002 can be applied. Public Health. 2018;165:6-8. doi: 10.1016/j.puhe.2018.07.005.

2. Correia MITD, Perman MI, Waitzberg DL. Hospital malnutrition in Latin America: a systematic review. Clin Nutr. 2017; 36(4): 958-967. doi: 10.1016 / j.clnu.2016.06.025.

3. Morán López JM, Enciso Izquierdo FJ, Luengo Pérez LM, Beneítez Moralejo B, Piedra León M, de Luis DA, Amado Señaris JA. Financial impact of disease-related malnutrition at 
the San Pedro de Alcántara hospital. Estimated cost savings associated to a specialized nutritional survey. Endocrinol Diabetes Nutr. 2017;64(8):446-50. doi: 10.1016/j. endinu.2017.05.004.

4. Ozkalkanli M, Ozkalkanli D, Katircioglu K, Savaci S. Comparison of tools for nutrition assessment and screening for predicting the development of complications in orthopedic surgery. Nutr Clin Pract. 2009;24(2):274-80. doi: 10.1177/0884533609332087.

5. Meira OMA. Aplicação do protocolo de risco nutricional (NRS 2002) aos dolentes internados no serviço de Endocrinologia do Centro Hospitalar do Porto - Hospital de Santo António [Tesis de Licenciatura]. Oporto: Universidade do Porto; 2010. p.51.

6. Neelemaat F, Meijers J, Kruizenga H, van Ballegooijen H, van Bokhorst-de van der Schueren M. Comparison of five malnutrition screening tools in one hospital inpatient sample. J Clin Nurs. 2011;20(15-16):2144-52. doi: 10.1111/j.13652702.2010.03667.

7. Kondrup J, Allison SP, Elia, M, Vellas B, Plauth M, Educational and Clinical Practice Committee, et al. ESPEN Guidelines for Nutrition Screening 2002. Clin Nutr. 2003;22(4):415-421. doi: 10.1016/S0261-5614(03)00098-0.

8. Kondrup J, Rasmussen HH, Hamberg O, Stanga Z; Ad Hoc ESPEN Working Group. Nutritional risk screening (NRS 2002): a new method based on an analysis of controlled clinical trials. Clin Nutr. 2003;22(3):321-336. doi: 10.1016/ S0261-5614(02)00214-5.

9. Gomes de Lima KV, Gomes de Lima L, Queiroz B, Evane M, Caraciolo de Almeida PA, Couto Santos EM, et al. Relação entre o instrumento de triagem nutricional (NRS-2002) y os métodos de avaliação nutricional objetiva em pacientes cirúrgicos do Recife (Pernambuco, Brasil). Nutr. Clín. diet. hosp. 2014; 34(3): 72-9. doi: 10.12873/343.

10. Ribeiro AMA, da Silva LL, Cardoso OG, Guedes LMH. Análise comparativa de diferentes métodos de triagem nutricional do paciente internado. Com. Ciências Saúde. 2010; 21 (4):331-42.

11. Raslan M, Gonzales MC, Gonçalves DMC, Paes-Barbosa FC, Cecconello I, Waitzberg DL. Aplicabilidade dos métodos de triagem nutricional no paciente hospitalizado. Ren. Nutr. 2008; 21(5):553-61.

12. Jaimes AE, Zúñiga TMG, Medina GA, Reyes BDY, Cruz CAB. Prevalence of nutritional risk in a hospital of second level in the Mexico State. Nutr clin diet hosp. 2016; 36(4):111-116. doi: $10.12873 / 364$ jaimes.

13. Carrasco LLE, Zavala GJC. Riesgo nutricional en servicios de hospitalización de medicina de un hospital general. Rev. Soc. Peruana Med. Interna. 2016; 29(2):53.

14. Arroyo-Sánchez AS, Aguilar LP, Obando BR, Sifuentes LC, Vrhunc Z, Avila LMJM. Cribado nutricional y su asociación con los resultados al alta hospitalaria. Rev. Soc. Peruana Med. Interna. 2015; 28(4):158-65.

15. Fontes D, Generoso SV, Toulson DCMI. Subjective global assessment: a reliable nutritional assessment tool to predict outcomes in critically ill patients. Clin Nutr. 2014;33(2):2915. doi: 10.1016/j.clnu.2013.05.004.

16. Zhang YH, Xie FY, Chen YW, Wang HX, Tian WX, Sun WG, et al. Evaluating the Nutritional Status of Oncology Patients and Its Association with Quality of Life. Biomed Environ Sci. 2018;31(9):637-44. doi: 10.3967/bes2018.088. PMID: 30369342.

17. Alvarez-Altamirano K, Delgadillo T, García-García A, Alatriste-Ortiz G, Fuchs-Tarlovsky V. Prevalence of nutritional risk evaluated with NRS-2002 in Mexican oncology population. Nutr Hosp. 2014;30(1),173-8. doi:10.3305/ nh.2014.30.1.7461.

18. Khalatbari-Soltani S, Marques-Vidal P. Impact of nutritional risk screening in hospitalized patients on management, outcome and costs: A retrospective study. Clin Nutr. 2016;35(6):1340-6. doi: 10.1016/j.clnu.2016.02.012.

19. Olivares J, Ayala L, Salas-Salvadó J, Muñiz MJ, Gamundí A, Martínez-Indart L, et al. Assessment of risk factors and test performance on malnutrition prevalence at admission using four different screening tools. Nutr Hosp. 2014;29(3):674-80. doi: 10.3305/nh.2014.29.3.7120.

20. Tevik K, Thürmer H, Husby M, de Soysa AK, Helvik AS. Nutritional risk screening in hospitalized patients with heart failure. Clin Nutr. 2015;34(2):257-64. doi: 10.1016/j. clnu.2014.03.014.

21. Schiesser M, Müller S, Kirchhoff P, Breitenstein S, Schäfer $\mathrm{M}$, Clavien P. Assessment of a novel screening score for nutritional risk in predicting complications in gastrointestinal surgery. Clin Nutr. 2008;27(4):565-70. doi: 10.1016/j. clnu.2008.01.010.

22. Javid Mishamandani Z, Norouzy A, Hashemian SM, Khoundabi B, Rezaeisadrabadi M, Safarian M, et al. Nutritional status of patients hospitalized in the intensive care unit: A comprehensive report from Iranian hospitals, 2018. J Crit Care. 2019; 54:151-8. doi: 10.1016/j.jcrc.2019.08.001.

23. Roncon NP, Moreira TTSL, Assis WF, Higino CC, Mourão L, Ferretti RL. Applicability of modified nutric, a nutritional risk assessment tool, in critical patients in an intensive care unit. Clin Nutr. 2018;37:S172. doi: 10.1016/j.clnu.2018.06.1626.

24. Brascher JMM, Peres WAF, Padilha PC. Use of the modified "Nutrition Risk in the critically ill" score and its association with the death of critically ill patients. Clin Nutr. ESPEN. 2019; 35: 162-6. doi: 10.1016/j.clnesp.2019.10.005.

25. Gunningberg L, Persson C, Åkerfeldt T, Stridsberg M, Swenne CL. Pre- and postoperative nutritional status and predictors for surgical-wound infections in elective orthopaedic and thoracic patients. Clin Nutr. ESPEN. 2008;3(3): e93-e101. doi: 10.1016/j.eclnm.2008.02.002.

26. Li XY, Yu K, Yang Y, Wang YF, Li RR, Li CW. Nutritional risk screening and clinical outcome assessment among patients with community-acquired infection: A multicenter study in Beijing teaching hospitals. Nutrition. 2016;32(10):1057-62. doi: 10.1016/j.nut.2016.02.020. 
27. Felder S, Lechtenboehmer C, Bally M, Fehr R, Deiss M, Faessler L, et al. Association of nutritional risk and adverse medical outcomes across different medical inpatient populations. Nutrition. 2015;31(11-12):1385-93. doi: 10.1016/j. nut.2015.06.007.

28. Vallejo KP, Martínez CM, Matos Adames AA, FuchsTarlovsky V, Nogales GCC, Paz RER, et al. Current clinical nutrition practices in critically ill patients in Latin America: a multinational observational study. Crit Care. 2017;21(1):227. doi: 10.1186/s13054-017-1805-z.

29. Bendavid I, Singer P, Theilla M, Themessl-Huber M, Sulz I, Mouhieddine M, et al. NutritionDay ICU: A 7 year worldwide prevalence study of nutrition practice in intensive care. Clin Nutr. 2017;36(4):1122-9. doi: 10.1016/j.clnu.2016.07.012.
30. Ridley EJ, Davies AR, Parke R, Bailey M, McArthur C, Gillanders L, et al. Supplemental parenteral nutrition in critically ill patients: A study protocol for a phase II randomised controlled trial. Trials. 2015;16:587.

31. Ridley EJ, Davies AR, Parke R, Bailey M, McArthur C, Gillanders L, et al. Supplemental parenteral nutrition versus usual care in critically ill adults: a pilot randomized controlled study. Crit Care. 2018;22(1):12. doi: 10.1186/s13054-0181939-7. 